\title{
Consequences of Aneuploidy in Sickness and in Health
}

Samuel D. Rutledge and Daniela Cimini ${ }^{\star}$

Department of Biological Sciences and Biocomplexity Institute, Virginia Tech, Blacksburg, VA 24061 - USA

Word count: 2,322

Keywords: aneuploidy, fitness, chromosome instability, adaptability, trisomy

${ }^{*}$ Corresponding author:

Daniela Cimini

Department of Biological Sciences \& VBI

1015 Life Science Circle

Blacksburg, VA 24061

USA

e-mail: cimini@vt.edu 


\begin{abstract}
A link between aneuploidy and miscarriage or cancer in humans has been known for a long time. However, only in recent years the development of experimental models of wholechromosome aneuploidy has allowed investigators to take a closer look at how aneuploidy affects individual cells. Collectively, recent studies using these models have shown that aneuploidy induces transcriptomic and proteomic changes, chromosomal instability, and adaptation. In this article, we discuss the findings from these recent studies and present current and emerging models on how aneuploidy may be deleterious in certain contexts, but beneficial in others.
\end{abstract}




\section{Introduction}

Fundamental to life is a cell's ability to accurately divide its genomic material evenly between its daughter cells. A number of different chromosome segregation errors can lead to inaccurate distribution of the genetic material to the daughter cells during cell division and cause defects such as chromosome rearrangements, gain/loss of defined genomic regions, and/or gain/loss of entire chromosomes (i.e., aneuploidy). All these defects can have dire consequences on the health of both individual cells and the organism in which they arise. In this article, we will specifically discuss the consequences of whole-chromosome aneuploidy, which is known to be a leading cause of miscarriage in humans, but is also found in certain healthy tissues, and is a common feature of cancer cells. Although the association between aneuploidy and miscarriages or cancer has been known for a long time, only in recent years a number of newly developed experimental models of aneuploidy have allowed a more thorough investigation and deeper understanding of how aneuploidy affects individual cells. These models consisted of various systems with defined extra chromosomes, including strains of haploid budding yeast carrying specific disomies, mouse embryonic fibroblasts (MEFs) carrying specific trisomies as a result of Robertsonian fusions, and trisomic or tetrasomic human cell lines generated via microcell-mediated chromosome transfer. Our discussion will focus on these recent studies in an attempt to highlight common themes and important differences.

\section{Effects of aneuploidy on gene expression and protein levels.}

Initial studies in haploid yeast strains carrying specific disomies $\left[1^{\bullet \bullet}\right]$ or MEFs carrying defined trisomies $\left[2^{\bullet}\right]$ reported an increased expression of genes on the aneuploid chromosome. A later study on a large panel of aneuploid yeast strains revealed that both the transcriptomic and the proteomic profiles scaled up with the aneuploidy [30*]. Proteomic changes in aneuploid yeast were also confirmed in a recent study $\left[4^{\circ}\right]$ and a direct correlation between 
chromosome copy number and gene expression levels was identified in several studies using aneuploid human cells $\left[5^{\circ}-7^{\circ}\right]$. Moreover, one of these studies also found changes at the proteomic level to correlate with the specific aneuploidy in human cells $\left[7^{\bullet \bullet}\right]$. Thus, these studies collectively highlighted a scale-up phenomenon by which the RNAs and the proteins corresponding to genes on the aneuploid chromosome(s) are present in higher amounts compared to those found in euploid controls.

However, one study also found that the levels of some proteins ( $25 \%)$ encoded on the aneuploid chromosome(s) were maintained at levels more similar to the diploid level, with protein kinases and subunits of protein complexes being the majority of those [ $\left.7^{\bullet \bullet}\right]$. Moreover, it was shown, both in yeast and human cells, that aneuploidy results in mis-regulation of a set of genes independent of the specific aneuploidy $\left[4^{\bullet}, 7^{\circ \bullet}\right]$. Indeed, at the protein level, human cells displayed downregulation of DNA and RNA metabolism pathways and upregulation of pathways linked to autophagy and lysosome function, vesicle transport, membrane synthesis, and carbohydrate and oxidative metabolic processes $\left[7^{\circ}\right]$. In a different study, transcriptomic analysis of a number of aneuploid cell lines identified downregulation of genes linked to DNA replication, transcription, and ribosomes and upregulation of genes linked to endoplasmic

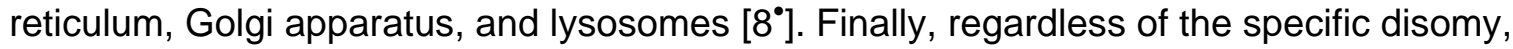
aneuploid budding yeast displayed a "gene expression signature" corresponding to upregulation

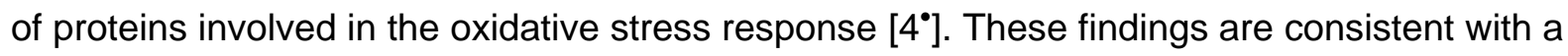
previous meta-analysis performed by Sheltzer and colleagues [9], who found that different aneuploidies arising in many different species, including yeast, plants, mice, and humans, produced certain consistent gene expression changes, independent of the aneuploidy and of the species. These changes consisted in the upregulation of genes involved in the response to stress and downregulation of genes associated with cell cycle and cell proliferation [9]. Yet, other investigators observed association in human cells between aneuploidy and upregulation of 
proteins involved in DNA metabolism (as opposed to the downregulation reported in [ $\left.7^{\circ *}\right]$ ) and growth $\left[10^{\circ}\right]$. Based on these observations, several groups of investigators have argued that aneuploidy induces a defined gene mis-expression pattern, possibly as a result of a physiological response to the stress caused by carrying an excess of hundreds-to-thousands genes. However, an alternative, not mutually exclusive, possibility is that genes on the aneuploid chromosome may act as regulators of genes on other chromosomes; this is consistent with studies performed on colorectal cancer cell lines with or without defined aneuploidies $\left[10^{\circ}, 11\right]$. In these studies, trisomy 7 and trisomy 13 were found to induce mis-

regulation of genes on chromosomes other than the aneuploid ones (in addition to those on the aneuploid chromosomes), but the genes mis-regulated in response to trisomy 7 were different than those mis-regulated in response to trisomy $13\left[10^{\circ}, 11\right]$.

Thus, the data available so far show that aneuploidy causes upregulation of genes carried by the additional chromosome(s), as well as mis-regulation of genes mapping on other chromosomes.

\section{Effects of aneuploidy on cell fitness and proliferation}

Given the long-known association between aneuploidy and disease, it would be hard to argue against the statement that aneuploidy is an undesirable trait. However, solid evidence on how aneuploidy affects cell physiology and proliferation has only emerged over the last decade. First, haploid yeast strains carrying defined aneuploidies were shown to display a G1 delay, reduced proliferation, and reduced ability to form colonies [1*0]. Subsequently, similar studies performed in MEFs carrying defined trisomies showed that aneuploid MEFs, similarly to aneuploid yeast, displayed impaired proliferation and impaired metabolism [2*0].

Given its generally negative effects on cell fitness and proliferation, it is surprising that aneuploidy is a physiological, and in some cases even necessary, condition in certain healthy 
tissues. For instance, aneuploidy is frequently found in hepatocytes of healthy human liver $\left[12^{\bullet}, 13\right]$. Perhaps more strikingly, during the development of the Drosophila rectum, papillar cells undergo endoreduplication/polyploidization and then re-enter mitosis $\left[14,15^{\circ}\right]$. These mitoses are highly error-prone and the cell population accumulates high levels of aneuploidy, but the suppression of pre-mitotic endocycles (i.e., the reduction of aneuploidy) leads to defective rectum development and reduced organismal tolerance for a high-salt diet [15]. Finally, the fact that aneuploidy is so commonly found in cancer cells $[16,17]$ and does not appear to interfere with their proliferation, suggests that under certain circumstance aneuploidy may, in fact, be beneficial.

\section{Effects of aneuploidy on chromosome stability}

The question of whether aneuploidy affects chromosome stability has been debated for a long time, with reports alternatively concluding that aneuploidy induces chromosome number instability (CIN) [18-20] or that it does not [21-23]. However, in recent years, studies in aneuploid yeast strains have unquestionably shown that aneuploidy causes genomic instability $\left[24^{\bullet \bullet}, 25^{\bullet \bullet}\right]$. Based on these results, a re-evaluation of the effects of aneuploidy on chromosome stability in human cells was warranted. In a recent study, amniocytes from aneuploid embryos were shown to display high rates of aneuploidy for chromosomes other than the constitutively aneuploid one [26]. In another study, both cancer cells and amniocytes carrying specific aneuploidies were shown to display high rates of mitotic chromosome mis-segregation in the form of anaphase lagging chromosomes (LCs) and chromosome number heterogeneity [27 ${ }^{\circ \bullet}$. LCs (chromosomes that lag behind at the spindle equator when all other chromosomes move to the poles during anaphase) are known to be the most common chromosome segregation defect seen in cancer cells $[22,28]$. These new findings $\left[27^{\circ \bullet}\right]$ now explain the correlation that had been previously identified between the degree of aneuploidy and the rates of LCs in cancer cells 
[29]. In addition to the chromosome number instability, these high rates of LCs also promote chromosome structural defects, given that LCs form micronuclei (MNi) upon mitotic exit [30,31] and that MNi have been shown to accumulate DNA damage and extensive chromosome rearrangements [31-33].

To conclude, recent data strongly support a role of aneuploidy in promoting chromosome mis-segregation and chromosomal/genomic instability.

\section{Aneuploidy and adaptability}

A plethora of data indicates that aneuploidy negatively affects cellular and organismal wellness. Indeed, aneuploidy (even mosaic) is a leading cause of miscarriage and congenital defects in humans [34]. Moreover, inducing aneuploidy in mouse models by mitotic checkpoint impairment can result in increased rates of or susceptibility to tumorigenesis [35,36]. Finally, as described above, aneuploidy typically impairs cell fitness and proliferation. However, a number of studies in single cell eukaryotes revealed that aneuploidy may confer a selective advantage under stressful environmental conditions. For instance, aneuploidy is associated with acquisition of antifungal resistance in Candida albicans [37,38]. Similarly, aneuploidy was shown to confer a selective advantage to budding yeast exposed to DNA damaging compounds [3*0]. In both cases, the aneuploidy resulted in overexpression of efflux pump genes, suggesting that aneuploidy may allow for rapid evolution of advantageous phenotypic traits. This conclusion is also supported by a previous study showing the emergence of aneuploid karyotypes that would result in alternative cytokinesis pathways in yeast strains lacking the MYO1 gene [39], as well as more recent studies showing the emergence of aneuploidy in response to telomerase insufficiency [40] or oxidative stress response deficiency [41 $\left.{ }^{\circ}\right]$.

A few examples of specific aneuploidies arising in response to environmental challenges also exist for mammalian cells. For example, loss of chromosome 16 in mouse hepatocytes was 
found to protect from hepatic injury [ $\left.42^{\circ}\right]$. In a different study, trisomy 7 , associated with overexpression of EGFR (encoded on chromosome 7), was shown to emerge in immortalized human colon epithelial cells cultured in serum-free media [43]. Emergence of trisomy 7 was also reported for human neural progenitor cells upon EGF withdrawal [44].

The studies described above suggest that aneuploidy can confer a selective advantage by specifically causing mis-expression of one or few genes on the aneuploid chromosome (Figure 1). Based on this, it has been proposed that environmental challenges could be used to select for specific "druggable" karyotypes/phenotypes [45]. However, it is possible that in other circumstances aneuploidy may act via a more general mechanism. Specifically, by causing chromosome mis-segregation and chromosomal/genomic instability $\left[24^{* *}, 25^{\circ *}, 26,27^{* *}\right]$, aneuploidy produces genetic heterogeneity in the cell population (Figure 2). This heterogeneity would then make the population adaptable to a broader spectrum of environmental challenges/conditions (Figure 2). This latter model can explain the prevalence and/or requirement of aneuploidy in certain healthy tissues, such as the human liver $\left[13,42^{\circ}\right]$ and the Drosophila rectum [15], that are more likely than other anatomical sites to be exposed to environmental toxins against which aneuploidy may confer protection/resistance. Moreover, such non-specific adaptability can also explain the widespread aneuploidy and CIN in cancer and the association between $\mathrm{CIN}$ and drug resistance in cancer cells $[46,47]$. Finally, this nonspecific adaptability model is also in agreement with our recent finding that both trisomy 7 and trisomy 13 confer a selective advantage to cancer cells cultured under several non-standard conditions, with no evidence for a link between a given aneuploidy and a specific condition (Rutledge, Cimini et al., unpublished).

The final question, then, is how does aneuploidy exactly affect cellular fitness? It has been previously proposed that aneuploid cells may be best fit to certain conditions that deviate from a "standard" culture/environmental condition (Figure 3, dashed yellow lines) [45]. However, 
another possibility is that aneuploid cells are never well-fit for any environmental condition, but can adapt to a broader range of conditions (Figure 3, solid yellow line), which allows them to survive in contexts that are too hostile for their euploid counterparts. Thus, although under standard environmental conditions the fitness of diploid cells (Figure 3, solid blue line) is substantially higher than that of aneuploid cells, as the environmental conditions deviate from the standard, aneuploid cells rapidly become better fit than diploid cells (Figure 1C, solid yellow line). This subpar broad fitness may be the result of two, not mutually exclusive, factors: first,

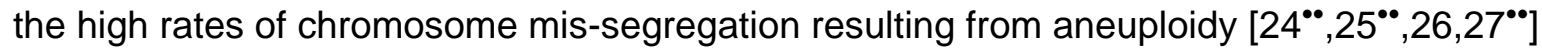
increase the karyotypic/genomic heterogeneity of the cell population (as shown in Figure 2), making the population adaptable to a wider range of conditions; second, the upregulation of stress response pathways caused by aneuploidy $\left[4^{\bullet}, 7^{\circ}\right]$ may "prime" aneuploid cells' response against environmental stresses and therefore allow for more rapid adaptation to changes in environmental conditions.

\section{Conclusions and Outlook}

Collectively, recent findings reveal a complex relationship between specific aneuploid karyotypes and the resulting phenotypes, indicating that the consequences of aneuploidy depend on numerous factors, including the specific chromosome being gained, the cell type in which the aneuploidy occurs, and the environmental context in which the aneuploid cell exists.

Despite the significant progress made in recent years in understanding the effects of aneuploidy on cell physiology, some big open questions still remain. One major question concerns the effects of chromosome loss/monosomy on cell function, but this question cannot be addressed with current model systems. Indeed, the yeast models of aneuploidy developed in recent years are based on haploid strains and chromosome loss would generate a nullisomy, which would be lethal. Similarly, the mammalian cell models are either from amniocentesis 
samples (where trisomy is the most common type of abnormality) or generated via microcellmediated chromosome transfer, thus exclusively consisting of hyperploid models. Therefore, new experimental models must be developed to specifically investigate the consequences of monosomy.

Another open question concerns the differences between different aneuploidies. Indeed, whereas several studies have focused on the commonalities between different aneuploidies, the data also clearly show that genes on the aneuploid chromosome are typically mis-expressed, thus indicating that there may be aneuploidy-specific effects/phenotypes that have not been fully characterized to date. This problem is not easy to dissect given the large number of genes whose expression is altered in any given aneuploidy. However, it seems reasonable to think that these aneuploidy-specific phenotypes may be particularly relevant to cancer, given that different cancers are known to accumulate specific aneuploidies [29]. Thus, understanding the aneuploidy-specific effects on the physiology of different cell types, will provide critical insight into the biology of cancer and potential tools for cancer therapy.

\section{Acknowledgements}

We would like to thank all the members of the Cimini lab for helpful discussions and critical reading of the manuscript. Work in the Cimini lab is supported by NSF grant MCB-1517506. 


\section{References.}

1. “Torres EM, Sokolsky T, Tucker CM, Chan LY, Boselli M, Dunham MJ, Amon A: Effects of aneuploidy on cellular physiology and cell division in haploid yeast. Science 2007, 317:916-924.

This was the first study looking at a large panel of aneuploid yeast strains. The authors observed a general overexpression of genes on the aneuploid chromosome. Moreover, they found that aneuploidy resulted in cell cycle delay, reduced proliferation, and reduced ability to form colonies.

2. “Williams BR, Prabhu VR, Hunter KE, Glazier CM, Whittaker CA, Housman DE, Amon A: Aneuploidy affects proliferation and spontaneous immortalization in mammalian cells. Science 2008, 322:703-709.

This is a seminal study on the effects of aneuploidy on mammalian cells. MEFs carrying defined trisomies were shown to display increased expression of genes on the aneuploid chromosome. Moreover, these aneuploid MEFs displayed impaired proliferation and impaired metabolism.

3. “Pavelka N, Rancati G, Zhu J, Bradford WD, Saraf A, Florens L, Sanderson BW, Hattem GL, Li R: Aneuploidy confers quantitative proteome changes and phenotypic variation in budding yeast. Nature 2010, 468:321-325.

This comprehensive study on a panel of aneuploid yeast strains showed that both the transcriptomic and the proteomic profiles scaled up with the aneuploidy. Moreover, aneuploidy was shown to confer selective advantage to budding yeast exposed to DNA damaging compounds, thus indicating that aneuploidy can promote adaptation.

4. 'Dephoure N, Hwang S, O'Sullivan C, Dodgson SE, Gygi SP, Amon A, Torres EM:

Quantitative proteomic analysis reveals posttranslational responses to aneuploidy in yeast. Elife 2014, 3:e03023.

Proteomic analysis showed an increase in proteins encoded on the aneuploid chromosomes. Moreover, there was upregulation of proteins involved in the oxidative stress response regardless of the aneuploidy, leading the authors to propose a "gene expression signature" associated with aneuploidy.

5. 'Ben-David U, Arad G, Weissbein U, Mandefro B, Maimon A, Golan-Lev T, Narwani K, Clark AT, Andrews PW, Benvenisty N, et al.: Aneuploidy induces profound changes in gene expression, proliferation and tumorigenicity of human pluripotent stem cells. Nat Commun 2014, 5:4825.

Transcriptomic analysis of human pluripotent stem cells with trisomy 12 showed an overall overexpression of genes located on chromosome 12. These cells also displayed increased proliferation and tumorigenicity, suggesting that aneuploidy can confere a proliferative advantage.

6. Gao C, Furge K, Koeman J, Dykema K, Su Y, Cutler ML, Werts A, Haak P, Vande Woude GF: Chromosome instability, chromosome transcriptome, and clonal evolution of tumor cell populations. Proc Natl Acad Sci U S A 2007, 104:8995-9000.

7. “-Stingele S, Stoehr G, Peplowska K, Cox J, Mann M, Storchova Z: Global analysis of genome, transcriptome and proteome reveals the response to aneuploidy in human cells. Mol Syst Biol 2012, 8:608.

This study showed a direct correlation between chromosome copy number and both transcript and protein levels. However, certain genes were consistently up- or down-regulated regardless of the specific aneuploidy. 
8. 'Dürrbaum M, Kuznetsova AY, Passerini V, Stingele S, Stoehr G, Storchova Z: Unique features of the transcriptional response to model aneuploidy in human cells. $B M C$ Genomics 2014, 15:139.

Proteomic analysis in aneuploid human cells showed downregulation of genes linked to DNA replication, transcription, and ribosomes and upregulation of genes linked to endoplasmic reticulum, Golgi apparatus, and lysosomes.

9. Sheltzer JM, Torres EM, Dunham MJ, Amon A: Transcriptional consequences of aneuploidy. Proc Natl Acad Sci U S A 2012, 109:12644-12649.

10. 'Gemoll T, Habermann JK, Becker S, Szymczak S, Upender MB, Bruch HP, Hellman U, Ried T, Auer G, Jornvall H, et al.: Chromosomal aneuploidy affects the global proteome equilibrium of colorectal cancer cells. Anal Cell Pathol (Amst) 2013, 36:149-161.

Using human colorectal cancer cells with defined aneuploidies, the authors found that a number of overexpressed genes mapped on chromosomes others than the aneuploid one(s).

11. Upender MB, Habermann JK, McShane LM, Korn EL, Barrett JC, Difilippantonio MJ, Ried T: Chromosome transfer induced aneuploidy results in complex dysregulation of the cellular transcriptome in immortalized and cancer cells. Cancer Res 2004, 64:69416949.

12. Duncan AW, Hanlon Newell AE, Smith L, Wilson EM, Olson SB, Thayer MJ, Strom SC, Grompe M: Frequent aneuploidy among normal human hepatocytes. Gastroenterology 2012, 142:25-28.

Using karyotyping and fluorescence in situ hybridization, the authors showed that human hepatocytes from healthy donors are highly aneuploid, suggesting that aneuploidy may be tolerated or even beneficial in some contexts.

13. Knouse KA, Wu J, Whittaker CA, Amon A: Single cell sequencing reveals low levels of aneuploidy across mammalian tissues. Proc Natl Acad Sci U S A 2014, 111:1340913414.

14. Fox DT, Gall JG, Spradling AC: Error-prone polyploid mitosis during normal Drosophila development. Genes Dev 2010, 24:2294-2302.

15. 'Schoenfelder KP, Montague RA, Paramore SV, Lennox AL, Mahowald AP, Fox DT: Indispensable pre-mitotic endocycles promote aneuploidy in the Drosophila rectum. Development 2014, 141:3551-3560.

This study shows that Drosophila papillar cells accumulate high levels of aneuploidy during development. Suppression of the mechanisms causing such aneuploidy leads to defective rectum development and reduced organismal tolerance for a high-salt diet. This exemplifies a case in which aneuploidy appears to be beneficial.

16. Cimini D: Merotelic kinetochore orientation, aneuploidy, and cancer. Biochim Biophys Acta 2008, 1786:32-40.

17. Weaver BA, Cleveland DW: Does aneuploidy cause cancer? Curr Opin Cell Biol 2006, 18:658-667.

18. Duesberg $P$, Rausch $C$, Rasnick D, Hehlmann R: Genetic instability of cancer cells is proportional to their degree of aneuploidy. Proc Natl Acad Sci U S A 1998, 95:1369213697. 
19. Reish O, Brosh N, Gobazov R, Rosenblat M, Libman V, Mashevich M: Sporadic aneuploidy in PHA-stimulated lymphocytes of Turner's syndrome patients. Chromosome Res 2006, 14:527-534.

20. Reish O, Regev M, Kanesky A, Girafi S, Mashevich M: Sporadic aneuploidy in PHAstimulated lymphocytes of trisomies 21, 18, and 13. Cytogenet Genome Res 2011, 133:184-189.

21. Lengauer C, Kinzler KW, Vogelstein B: Genetic instability in colorectal cancers. Nature 1997, 386:623-627.

22. Thompson SL, Compton DA: Examining the link between chromosomal instability and aneuploidy in human cells. J Cell Biol 2008, 180:665-672.

23. Valind $A$, Jin $Y$, Baldetorp B, Gisselsson D: Whole chromosome gain does not in itself confer cancer-like chromosomal instability. Proc Natl Acad Sci U S A 2013, 110:21119-21123.

24. “-Sheltzer JM, Blank HM, Pfau SJ, Tange Y, George BM, Humpton TJ, Brito IL, Hiraoka Y, Niwa O, Amon A: Aneuploidy drives genomic instability in yeast. Science 2011, 333:1026-1030.

By analyzing a large panel of aneuploid yeast strains, this study unquestionably showed that aneuploidy causes genomic instability in yeast.

25. • Zhu J, Pavelka N, Bradford WD, Rancati G, Li R: Karyotypic determinants of chromosome instability in aneuploid budding yeast. PLoS Genet 2012, 8:e1002719. Using a large panel of yeast strains with various degrees of aneuploidy, this study showed that aneuploidy causes CIN and that the degree of CIN varies with the degree of aneuploidy as well as the entity of the aneuploidy.

26. Biron-Shental T, Liberman M, Sharvit M, Sukenik-Halevy R, Amiel A: Amniocytes from aneuploidy embryos have enhanced random aneuploidy and signs of senescence - Can these findings be related to medical problems? Gene 2015.

27. “Nicholson JM, Macedo JC, Mattingly AJ, Wangsa D, Camps J, Lima V, Gomes AM, Doria $\mathrm{S}$, Ried T, Logarinho E, et al.: Chromosome mis-segregation and cytokinesis failure in trisomic human cells. Elife 2015, 4.

This study addressed the long-debated question of whether aneuploidy causes CIN. The authors showed that both human cancer cells and amniocytes carrying specific aneuploidies display high rates of LCs and karyotypic heterogeneity, thus providing strong evidence for a link between aneuploidy and CIN in human cells.

28. Bakhoum SF, Silkworth WT, Nardi IK, Nicholson JM, Compton DA, Cimini D: The mitotic origin of chromosomal instability. Curr Biol 2014, 24:R148-149.

29. Nicholson JM, Cimini D: Cancer karyotypes: survival of the fittest. Front Oncol2013, 3:148.

30. Cimini D, Fioravanti D, Salmon ED, Degrassi F: Merotelic kinetochore orientation versus chromosome mono-orientation in the origin of lagging chromosomes in human primary cells. J Cell Sci 2002, 115:507-515.

31. Crasta K, Ganem NJ, Dagher R, Lantermann AB, Ivanova EV, Pan Y, Nezi L, Protopopov A, Chowdhury D, Pellman D: DNA breaks and chromosome pulverization from errors in mitosis. Nature 2012, 482:53-58. 
32. Terradas M, Martin M, Hernandez L, Tusell L, Genesca A: Nuclear envelope defects impede a proper response to micronuclear DNA lesions. Mutat Res 2012, 729:3540.

33. Zhang CZ, Spektor A, Cornils H, Francis JM, Jackson EK, Liu S, Meyerson M, Pellman D: Chromothripsis from DNA damage in micronuclei. Nature 2015, 522:179-184.

34. Nagaoka SI, Hassold TJ, Hunt PA: Human aneuploidy: mechanisms and new insights into an age-old problem. Nat Rev Genet 2012, 13:493-504.

35. Kops GJ, Weaver BA, Cleveland DW: On the road to cancer: aneuploidy and the mitotic checkpoint. Nat Rev Cancer 2005, 5:773-785.

36. Weaver BA, Cleveland DW: Aneuploidy: instigator and inhibitor of tumorigenesis. Cancer Res 2007, 67:10103-10105.

37. Selmecki A, Forche A, Berman J: Aneuploidy and isochromosome formation in drugresistant Candida albicans. Science 2006, 313:367-370.

38. Selmecki AM, Dulmage K, Cowen LE, Anderson JB, Berman J: Acquisition of aneuploidy provides increased fitness during the evolution of antifungal drug resistance. PLoS Genet 2009, 5:e1000705.

39. 'Rancati G, Pavelka N, Fleharty B, Noll A, Trimble R, Walton K, Perera A, StaehlingHampton K, Seidel CW, Li R: Aneuploidy underlies rapid adaptive evolution of yeast cells deprived of a conserved cytokinesis motor. Cell 2008, 135:879-893.

This study showed the emergence of aneuploid karyotypes that would result in alternative cytokinesis pathways in yeast strains lacking the essential gene MYO1. This study represents a clear example of how aneuploidy can favor adaptation.

40. Millet C, Ausiannikava D, Le Bihan T, Granneman S, Makovets S: Cell populations can use aneuploidy to survive telomerase insufficiency. Nat Commun 2015, 6:8664.

41. 'Kaya A, Gerashchenko MV, Seim I, Labarre J, Toledano MB, Gladyshev VN: Adaptive aneuploidy protects against thiol peroxidase deficiency by increasing respiration via key mitochondrial proteins. Proc Natl Acad Sci U S A 2015, 112:10685-10690.

This study showed that survival of yeast strains deleted of all eight thiol peroxidase genes depended on the acquisition of an extra copy of chromosome XI, thus representing an important example of aneuploidy-dependent adaptation.

42. 'Duncan AW, Hanlon Newell AE, Bi W, Finegold MJ, Olson SB, Beaudet AL, Grompe M: Aneuploidy as a mechanism for stress-induced liver adaptation. J Clin Invest 2012, 122:3307-3315.

This study showed that aneuploidy could protect mouse models from liver damage. Thus, this represents an example of aneuploidy-induced adaptation within a multicellular organism.

43. Ly P, Eskiocak U, Kim SB, Roig AI, Hight SK, Lulla DR, Zou YS, Batten K, Wright WE, Shay JW: Characterization of aneuploid populations with trisomy 7 and 20 derived from diploid human colonic epithelial cells. Neoplasia 2011, 13:348-357.

44. Sareen D, McMillan E, Ebert AD, Shelley BC, Johnson JA, Meisner LF, Svendsen CN: Chromosome 7 and 19 trisomy in cultured human neural progenitor cells. PLOS One 2009, 4:e7630.

45. Chen G, Mulla WA, Kucharavy A, Tsai HJ, Rubinstein B, Conkright J, McCroskey S, Bradford WD, Weems L, Haug JS, et al.: Targeting the adaptability of heterogeneous aneuploids. Cell 2015, 160:771-784. 
46. Lee AJ, Endesfelder D, Rowan AJ, Walther A, Birkbak NJ, Futreal PA, Downward J, Szallasi Z, Tomlinson IP, Howell M, et al.: Chromosomal instability confers intrinsic multidrug resistance. Cancer Res 2011, 71:1858-1870.

47. Swanton C, Nicke B, Schuett M, Eklund AC, Ng C, Li Q, Hardcastle T, Lee A, Roy R, East $\mathrm{P}$, et al.: Chromosomal instability determines taxane response. Proc Natl Acad Sci U S A 2009, 106:8671-8676. 


\section{Figure legends}

Figure 1. Adaptability to environmental challenge conferred by overexpression of one or few specific gene(s) on the aneuploid chromosome. The lightning bolt represents a challenge or change in environmental conditions. A specific aneuploidy (n) can confer resistance to a given challenge that would kill a euploid cell $(2 \mathrm{~N}=$ diploid, mammals; $\mathrm{N}=$ haploid, yeast). The circles represent individual cells.

Figure 2. Adaptability conferred by the karyotypic/phenotypic heterogeneity caused by aneuploidy. By promoting chromosome mis-segregation/CIN, aneuploidy results in karyotypic (and thus phenotypic) heterogeneity within the cell population. The different karyotypes/phenotypes present within such heterogeneous population may be adapted/resistant to defined environmental conditions. Once these cells are exposed to certain challenges/environmental changes, individual aneuploid cells (the ones with the fittest karyotype/phenotype) may survive. These cells, in turn, can generate new heterogeneous cell population due to the intrinsic CIN associated with aneuploidy. The lightning bolts represent challenges or changes in environmental conditions and the different colors refer to different challenges/changes. The large circles represent individual cells. The small colored circles represent extra copies of various chromosomes.

Figure 3. Relationship between karyotype and cellular fitness. Diploid/haploid cells (solid blue line) are well adapted to certain "standard" conditions, but their fitness rapidly decreases as the environmental conditions deviate from the standard. It has been previously proposed that aneuploid cells may display optimal fitness under conditions that deviate from the standard conditions (dashed yellow lines). An alternative possibility is that aneuploid cells display suboptimal fitness under standard conditions, but their fitness extends over a broader range of environmental conditions (solid yellow line). 
Figure 1
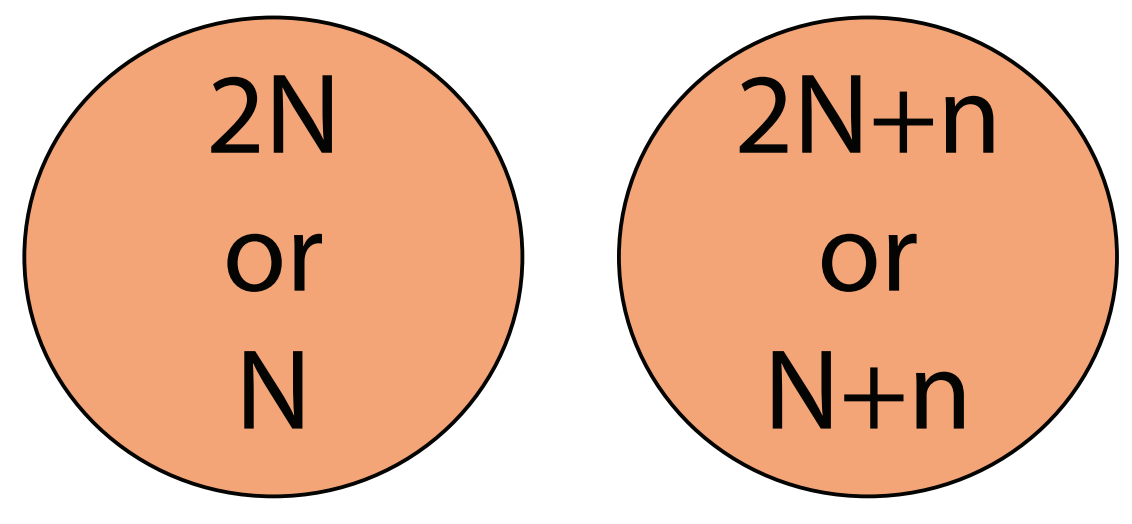

Challenge $X$

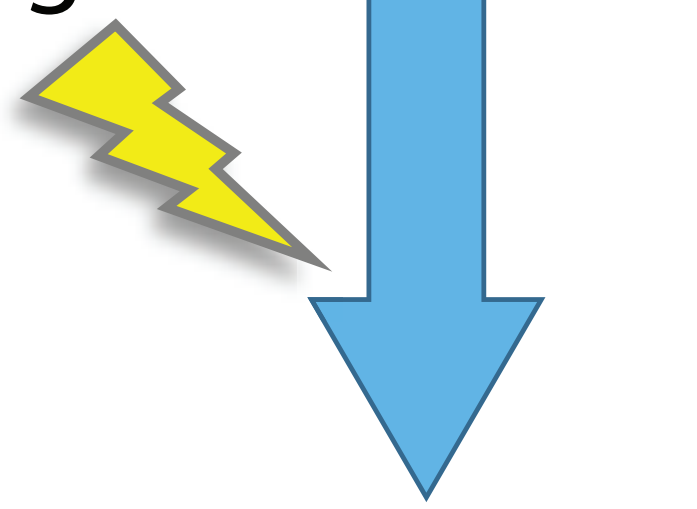

Challenge $\mathrm{X}$
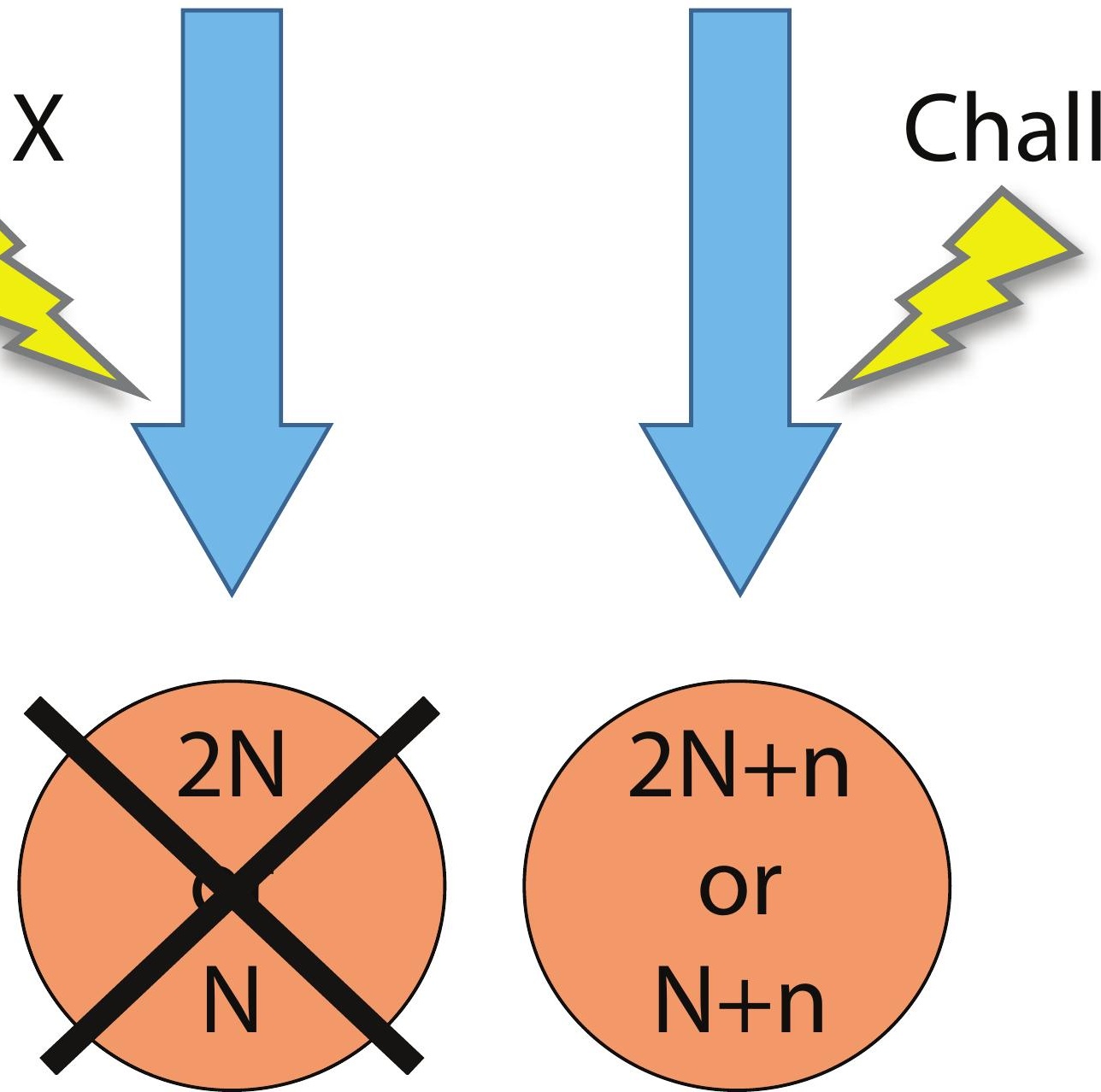
